\title{
Estado de las poblaciones del Rayador (Rynchops niger) en Chile
}

\section{Population status of the Black skimmer (Rynchops niger) in Chile}

\section{Roberto F. Thomson ${ }^{1, *}$, María Angélica Vukasovic ${ }^{1}$ \& Cristián F. Estades $^{1}$}

'Laboratorio de Ecología de Vida Silvestre, Facultad de Ciencias Forestales y de la Conservación de la Naturaleza, Universidad de Chile. Santa Rosa 11315, La Pintana, Región Metropolitana, Chile.

*E-mail: rthomson@uchile.cl

\section{RESUMEN}

El rayador (Rynchops niger) visita las costas de Chile durante los veranos australes, observándose en bandadas de distinto tamaño principalmente en humedales costeros. Por medio del uso de una base de datos de observaciones de ciencia ciudadana junto a nuestros monitoreos de largo plazo, estimamos que el tamaño poblacional promedio para la especie en Chile alcanzó los 10.227 individuos entre 2014 y 2018. Tres sitios (estuarios de los ríos Mataquito, Itata, y Tubul) congregaron a más del $50 \%$ de la población. El análisis de la abundancia relativa a través del año para cuatro macrozonas geográficas reflejaría el movimiento de la especie en las costas de Chile. La zona Norte muestra un peak de abundancia en noviembre con la llegada de individuos, no siendo tan clara su estrategia de retorno. Las zonas Centro-Norte y Centro del país muestran dos peaks en la llegada y partida de individuos, sugiriendo un uso más bien de tránsito. La existencia de un solo peak en el mes de febrero, además de las mayores concentraciones de individuos, indicarían que las zonas Centro-Sur y Sur del país serían potencialmente el destino para gran parte de la población de rayadores. El seguimiento de individuos por medio de radiotelemetría permitió detectar movimiento de individuos entre una red de humedales costeros. Estos desplazamientos ocurrirían frecuentemente a lo largo de toda la temporada y tanto en dirección norte como hacia el sur. Este trabajo presenta información relevante para la evaluación del estado de la especie en Chile, país que potencialmente alberga un porcentaje significativo de la población de la subespecie cinerascens durante el período no reproductivo.

Palabras clave: ciencia ciudadana, cinerascens, eBird, estuarios, tamaño poblacional, telemetría.

\section{ABSTRACT}

Black skimmers (Rynchops niger) visit the coast of Chile during the Southern summer, being observed in flocks of different sizes, mainly in coastal wetlands. Through the joint use of a database of citizen science observations together with our long-term monitoring, we estimate that the population size for the species in Chile reaches 10,227 individuals. Three sites congregate more than $50 \%$ of the population, these sites are the estuaries of Mataquito and Itata river, and Tubul. The analysis of the relative abundance throughout the year for four geographic macrozones would reflect the movement of the species on the coasts of Chile. The Northern zone shows an abundance peak in November with the arrival of individuals, yet its return strategy keeps not being so clear. The Central-North and Central zones of the country show two peaks in the arrival and departure of individuals, suggesting a rather transit use. The existence of a single peak in February, in addition to the highest concentrations of individuals, would indicate that the Central-Southern and Southern areas of the country would potentially be the destination for a large part of the population of black skimmers. The radiotelemetry monitoring of individuals allowed the detection of movement of individuals between a network of coastal wetlands. These displacements would occur frequently throughout the season and both north and southbound. This study provides relevant information for the status of the species in Chile, a country that is habitat for a potentially significant proportion of the cinerascens subspecies during the non-breeding period.

Keywords: cinerascens, citizen science, eBird, estuaries, population size, telemetry. 


\section{INTRODUCCIÓN}

La costa occidental de Sudamérica recibe cada verano austral la visita de un gran número de aves migratorias (Sallaberry et al. 1996). Entre éstas, una de las especies más conspicuas es el rayador (Rynchops niger), ave que se reproduce en ríos tropicales de Sudamérica y que llega a estuarios de Chile central en grandes números (Estades et al. 2017, Groom 1992). A nivel mundial, R. niger tiene tres subespecies. En Norteamérica, hasta el Caribe, se observa la subespecie niger. La subespecie sudamericana intercedens está presente en el Este de Brasil y Noreste de Argentina (Davenport et al. 2016). Finalmente, la subespecie amazónica cinerascens está presente en el Norte y Oeste de Sudamérica. Esta es la subespecie que visita Chile, aunque también es posible observar algunos individuos de la subespecie intercedens, que migra después de la época reproductiva a la zona del Pantanal y la costa Atlántica (Vieira et al. 2018). La subespecie intercedens se distingue de cinerascens por una marcada banda distal y partes interiores de las alas de color blanco (Wetmore 1944).

Uno de los aspectos más destacables de esta especie es su especialización en la captura de pequeños peces cerca de la superficie del agua, principalmente de noche, conducta que se asocia a dos adaptaciones anatómicas notables como son su mandíbula inferior elongada en forma de cuchilla (Zusi 1962) y sus pupilas rasgadas, con mayor proporción de bastoncitos en sus retinas para una mejor visión nocturna (Zusi \& Bridge 1981).

Esta alta especialización trófica se traduce también en una gran especialización de hábitat. El rayador depende fuertemente de aguas calmas y poco profundas para su alimentación, las que encuentra en las zonas estuarinas de varios ríos de Chile central, donde se agrega desde mediados de noviembre hasta el mes de mayo (Estades \& Vukasovic 2013). Entre estos sitios sobresale el estuario del río Itata $\left(36^{\circ} 23^{\prime} \mathrm{S}, 72^{\circ} 51^{\prime} \mathrm{W}\right)$, el que concentra valores que superarían el criterio del $1 \%$ de la población mundial de la especie para calificar como sitio Ramsar (Estades \& Vukasovic 2013).

Más allá del caso de algunos sitios específicos, el estado general de las poblaciones de rayador en Chile no está bien entendido, particularmente la distribución de las poblaciones entre los distintos sitios de agregación de la especie. Para avanzar en cerrar esta brecha de conocimiento llevamos a cabo un estudio en el que examinamos información poblacional de la especie proveniente de distintas fuentes. Particularmente, estimamos el tamaño de la población que migra a Chile, utilizando una base de datos de ciencia ciudadana junto a nuestros datos de censos de la especie en humedales costeros de la zona central de Chile. Además, para contribuir a entender los desplazamientos y variaciones en el número de individuos observados entre una serie de estuarios de la zona central, complementamos nuestro análisis con el marcaje y radio-seguimiento de individuos.

\section{MATERIALES Y MÉTODOS}

\section{ÁREA DE ESTUDIO}

El estudio se desarrolló en dos áreas geográficas de escalas diferentes. En primer lugar, para la estimación de la abundancia y distribución de la especie en Chile, utilizamos información para todo el territorio nacional. Por otro lado, para el análisis de la redistribución de individuos entre humedales de la zona central de Chile, la zona de estudio abarcó la costa entre los $32^{\circ} 54^{\prime} \mathrm{S}$ y $37^{\circ} 14^{\prime} \mathrm{S}$. Esta región se caracteriza por la alta productividad del mar costero, sostenida por el sistema de la corriente de Humboldt (Daneri et al. 2000). Aunque con algún gradiente latitudinal, en general la zona presenta un clima templado con veranos secos, con ocurrencia de lluvias estivales infrecuentes en la zona sur (Hajek \& Di Castri 1975). Dentro de este rango geográfico es posible encontrar varios estuarios de distinta extensión e influencia antrópica, destacando por la congregación de aves migratorias durante el verano austral el estuario del río Maipo y el del río Itata (Soazo et al. 2009; González et al. 2011).

FUENTES DE INFORMACIÓN SOBRE ABUNDANCIA Y DISTRIBUCIÓN Con el fin de estimar la abundancia y distribución de la especie en Chile, recurrimos a dos fuentes de información: la base de datos eBird (Sullivan et al. 2014) e información de censos de un programa de monitoreo de largo plazo (Estades \& Vukasovic 2013) llevado a cabo por el Laboratorio de Ecología de Vida Silvestre de la Universidad de Chile. Restringimos el análisis para un período de 5 años (2014 a 2018).

\section{TRATAMIENTO DE DATOS DE EBIRD}

La lista de registros de rayador facilitada por los administradores de eBird (ver agradecimientos) se sometió a una revisión exhaustiva para eliminar las entradas repetidas (i.e. mismo grupo de aves reportado por dos o más personas) y los registros que sólo reportan la presencia de la especie (i.e. marcada con una " $x$ "). Con este proceso, la lista se redujo de 3.730 a 2.223 registros únicos de la especie en Chile para el período 2014-2018. Además, debido a que los voluntarios ingresan sus observaciones de forma libre en la plataforma eBird, existen sitios con más de una denominación. Por esta razón, realizamos un análisis de la equivalencia de nombres de las localidades, fusionando aquellos que evidentemente se referían al mismo lugar. Al final de este procedimiento contabilizamos una lista total de 122 sitios, abarcando desde 
la desembocadura del río Lluta (18 $25^{\prime} \mathrm{S} 70^{\circ} 19^{\prime} \mathrm{W}$, Comuna de Arica) hasta bahía Pullao (42 $28^{\prime} \mathrm{S} 73^{\circ} 40^{\prime} \mathrm{W}$, Comuna de Castro).

Puesto que los registros proporcionados por eBird representan aquellos donde se observó al menos un individuo de la especie, para poder calcular la abundancia promedio de rayadores, determinamos desde la página web de eBird el número total de "listas de especies" (registros del total de aves y especies observadas en un momento dado por un observador) para cada uno de los 122 sitios durante el período de estudio. Asumimos que la diferencia entre el total de listas para un sitio y el número de registros que incluyen la especie se trataba de datos con valor 0 para el rayador. Así, con estos registros calculamos el promedio efectivo de individuos observados en cada sitio.

\section{Censos}

Los datos censales utilizados provienen de un programa de largo plazo de siete humedales costeros que involucra 10 (Itata) y 8 campañas anuales (Topocalma, Mataquito, Reloca, Laraquete, Carampangue, Tubul). Los censos representan el recuento completo de las aves acuáticas presentes en la zona estuarina de los mencionados ríos. Todos los censos se realizan con telescopio desde puntos fijos de observación (véanse detalles en Estades \& Vukasovic 2013). Con el fin de integrar estos datos con los provenientes de eBird, y elaborar diagramas mensuales de abundancia, transformamos los 10 u 8 registros anuales a valores mensuales mediante una interpolación temporal lineal (Acuña et al. 2019).

\section{INTEGRACIÓN Y ANÁLISIS DE DATOS POBLACIONALES}

Con el fin de establecer la abundancia y distribución de la especie a nivel nacional, compilamos los datos disponibles sobre abundancia promedio de la especie para cada sitio donde ésta se ha reportado durante el período de estudio. Para el caso de los sitios censados como parte de nuestro programa de monitoreo de largo plazo, estos datos fueron usados en reemplazo de los de eBird, debido a que se obtienen con un protocolo estándar, con los mismos observadores durante todo el período, y representan una base de datos de mucho mayor tamaño. Por ejemplo, para la desembocadura del río Itata, cada una de las 10 campañas anuales se compone de 3 días de censo, con dos censos por día (mañana y tarde, Estades \& Vukasovic 2013). Por lo tanto, las estimaciones poblacionales para este sitio se hicieron sobre $10 \times 6 \times 5=$ 300 censos para el período 2014-2018, mientras que, para el mismo período eBird reporta 48 listas, de las cuales sólo dos registran la especie.

Aunque para describir el cambio de abundancia de la especie a lo largo del año utilizamos todos los datos disponibles, para el cálculo de la abundancia total promedio del rayador en Chile, usamos sólo la información entre noviembre y abril, que es el período en que la especie concentra su presencia en el país.

\section{Captura y marcaje de individuos}

Mediante el uso de dos redes de niebla de $18 \mathrm{~m}$ de longitud y de un mesh de $127 \mathrm{~mm}$ (Avinet, USA), ubicadas sobre el curso del agua utilizando parantes de $6 \mathrm{~m}$ de altura, capturamos 10 individuos de rayador. Las capturas se realizaron en el estuario del río Itata $\left(36^{\circ} 23^{\prime} \mathrm{S} 75^{\circ} 12^{\prime} \mathrm{W}\right)$, durante noches sin luna, entre los días 28 de enero al 02 de febrero del año 2017. Los individuos capturados fueron marcados con anillos metálicos proporcionados por la oficina nacional de anillamiento (SNAA, SAG, Gobierno de Chile). Además, a estos individuos se les adosó un transmisor de radio frecuencia TXC-011B (Telenax Inc., México), por medio de un arnés hecho con cinta de teflón de 0.1 pulgadas (BallyRibbon Mills, Pennsylvania, USA), sumando un total de $10 \mathrm{~g}$ de masa. Las capturas fueron realizadas bajo el permiso $7237 / 2016$, emitido a nombre de MAV y RT.

\section{Monitoreo de RADIOTELEMETRÍA}

Durante los siguientes 12 meses desde la captura y marcaje de los individuos realizamos 10 campañas de detección de las aves en ocho estuarios de la zona centro sur de Chile, entre las latitudes $32^{\circ} 54^{\prime} \mathrm{S}$ y $37^{\circ} 14^{\prime} \mathrm{S}$, con el fin de detectar movimientos de aves entre áreas de concentración. Los sitios visitados fueron los estuarios de los ríos Aconcagua, Maipo, Topocalma, Mataquito, Reloca, Itata, Laraquete, Carampangue y Tubul (Fig. 2). Dos equipos de investigadores, utilizando receptores de telemetría R-1000 (COM-SPEC, California, USA) y antenas Yagi de 3 elementos, monitorearon la presencia de individuos con transmisores: un equipo monitoreó los estuarios de los ríos Aconcagua y Maipo su primer día, Topocalma el segundo día, Reloca el tercer día, para finalizar con el estuario del río Itata. El segundo grupo buscó la presencia de individuos con transmisores en los estuarios de los ríos Laraquete, Carampangue y Tubul en tres días consecutivos, y repitiéndose nuevamente la búsqueda durante los siguientes tres días.

\section{RESULTADOS}

Los datos analizados indican que, entre los meses noviembre a abril del período de estudio, la población promedio de rayadores en Chile fue de 10.227 individuos ( \pm 3.222 , i.c. $95 \%$, g.l.=4). Los tres sitios con mayor concentración de la especie fueron los estuarios de los ríos Tubul (37¹3's, 
$\left.73^{\circ} 26^{\prime} \mathrm{W}\right)$, Itata $\left(36^{\circ} 23^{\prime} \mathrm{S}, 73^{\circ} 51^{\prime} \mathrm{W}\right)$ y Mataquito $\left(35^{\circ} 07^{\prime} \mathrm{S}\right.$, $72^{\circ} 10^{\prime} \mathrm{W}$ ), con promedios de $2.973,1.757$ y 1.354 individuos, respectivamente. Sólo estos tres humedales concentraron el $59,5 \%$ del total de la población de la especie en el país. Durante el período estudiado, el $99,91 \%$ de los individuos registrados fueron observados en sitios costeros, siendo las únicas dos excepciones el lago Peñuelas (330.'S, $71^{\circ} 31^{\prime} \mathrm{W}$ )

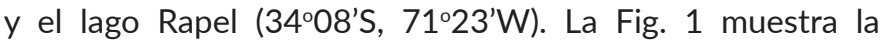
distribución de los distintos sitios con avistamientos de la especie en Chile. Adicionalmente, el Material Suplementario S1 contiene más detalles sobre los sitios y las poblaciones registradas en ellos. La base de datos de eBird sólo registra una observación de rayador fuera de esta zona geográfica, que corresponde a un individuo detectado en la localidad de Puerto Natales (514ㄱ'S, 72³0'W) en marzo de 2014.

En la Fig. 1 también se observa la distribución temporal del rayador en cinco macrozonas del país. En la zona Norte (Arica y Parinacota, Tarapacá y Antofagasta) existió una clara concentración de los registros durante el mes de noviembre, coincidente con la llegada de la especie al país. En las zonas Centro-Norte (Atacama y Coquimbo) y Centro (Valparaíso, Región Metropolitana y O'Higgins) se observaron dos máximos, en los meses de noviembre y de febrero. Finalmente, en las zonas Centro-Sur (Maule, Nuuble y Bío-bío) y Sur (Araucanía, Los Ríos y Los Lagos), la especie alcanzó sus máximos poblacionales durante el mes de febrero.

El mes de agosto fue el que presentó la menor abundancia de la especie en el país, con sólo un 1,2\% de la población observada entre noviembre y abril. Como ejemplo, durante este mes en el humedal de Tubul se registró un promedio (EE) de $14,8(5,4)$ individuos.

El monitoreo con radiotelemetría permitió registrar la presencia de 8 de los 10 individuos marcados durante el tiempo en que se desarrolló el seguimiento. Los individuos KK00402 y KK00406 no fueron detectados con posterioridad a su liberación. El transmisor KK00407 sólo fue registrado en el área de captura por 8 días. Uno de los transmisores (KK00401) fue recuperado en el sitio de captura con el arnés cortado 2 meses después de la instalación, y es posible que el individuo KK00409 haya sido depredado ya que su señal provenía desde un bosque aledaño a la ribera del río Itata. El individuo KK00410 sólo fue registrado en Itata hasta 2 meses después de su captura. Los individuos KK00403 y KK00405 fueron observados en 5 ocasiones y en 4 sitios distintos (Fig. 2). KKO0403 fue el único individuo observado en el estuario del río Aconcagua, el más septentrional de los estuarios monitoreados. Tres individuos (KK00403, KK00405 y KK00408) visitaron el estuario del río Reloca en, al menos, una ocasión (Fig. 2). Finalmente, el individuo KK00404, fue registrado en el estuario del río Itata en noviembre de 2017, coincidiendo con la llegada de la especie al país desde sus zonas de reproducción.

\section{DISCUSIÓN}

Aunque no es descartable que nuestra revisión haya pasado por alto algunos sitios con presencia de la especie, estamos confiados que todos los humedales relevantes para la especie fueron cubiertos por nuestro análisis. Por ejemplo, una revisión de datos más recientes de eBird no dan cuenta de la existencia de algún sitio importante no incluido en la presente compilación. Como sea, cualquier omisión representa una potencial subestimación de la población de la especie que visita Chile. El hecho de que este estudio esté basado en gran medida en datos provistos por observadores voluntarios plantea una incertidumbre en relación a la calidad de los datos. Sin embargo, la evidencia muestra que cuando los datos registrados son numerosos, como es crecientemente el caso de Chile (con más de 1.600.000 datos), iniciativas de ciencia ciudadana, como eBird pueden proveer información valiosa para la estimación de tamaños poblacionales y sus tendencias (Horns et al. 2018).

No existe claridad acerca del tamaño de la población global de R. n. cinerascens (Vieira et al. 2018), existiendo principalmente datos de ocurrencia y reportes del número de individuos observados en unos pocos sitios. Las mayores concentraciones de rayadores de esta subespecie se han reportado fuera de la temporada reproductiva en sitios de alta productividad, como estuarios, bahías y lagunas costeras, no superando los 4.000 individuos (Viera et al. 2018). Dado lo anterior, es posible que los más de 10.000 individuos que visitan Chile cada año representen un porcentaje importante del total de esta población.

Aunque los registros de eBird no diferencian entre las dos subespecies que visitan el país, en base a nuestras observaciones de campo estimamos que menos del $1 \%$ de los individuos correspondían a la subespecie intercedens.

Con la excepción de algunos visitantes ocasionales en la región de Magallanes (Clark 1986), los datos muestran que esta especie migratoria se distribuye en Chile sólo hasta el norte de la isla de Chiloé (Fig. 1). El porqué no continúa hasta más al sur no es evidente, pero al menos es posible plantear dos hipótesis no excluyentes entre sí. En primer lugar, este patrón de distribución sería esperable si la abundancia relativa de ambientes apropiados para la especie disminuye hacia el sur, lo que en conjunto con el aumento evidente del costo de desplazamiento hasta mayores latitudes haría que existiera un punto, eventualmente Chiloé, más allá del cual resulta ineficiente desplazarse. Por otro lado, el tipo especializado de 
alimentación del rayador hace que el viento excesivo afecte sus posibilidades de forrajeo (Burger 1982, Tomkins 1951). La inestabilidad creciente de las condiciones meteorológicas hacia el sur provoca un aumento en la frecuencia e intensidad de los vientos, lo que también podría contribuir a que la especie no avance más allá de Chiloé.

Otros factores que explican la distribución de la especie pueden tener un origen más antrópico. Por ejemplo, Estades et al. (2012) observaron que la abundancia registrada de la especie en humedales costeros de Chile se asociaba positivamente a la superficie de barras de arena aisladas, lo que sugeriría que la especie podría seleccionar estuarios con áreas de descanso con acceso reducido para perros. Un estudio realizado con la gaviota de Franklin (Leucophaeus pipixcan) y el gaviotín elegante (Thalaseus elegans) en el humedal de Carampangue identificó a los perros como el principal agente de perturbación para estas aves (Espinoza 2018).

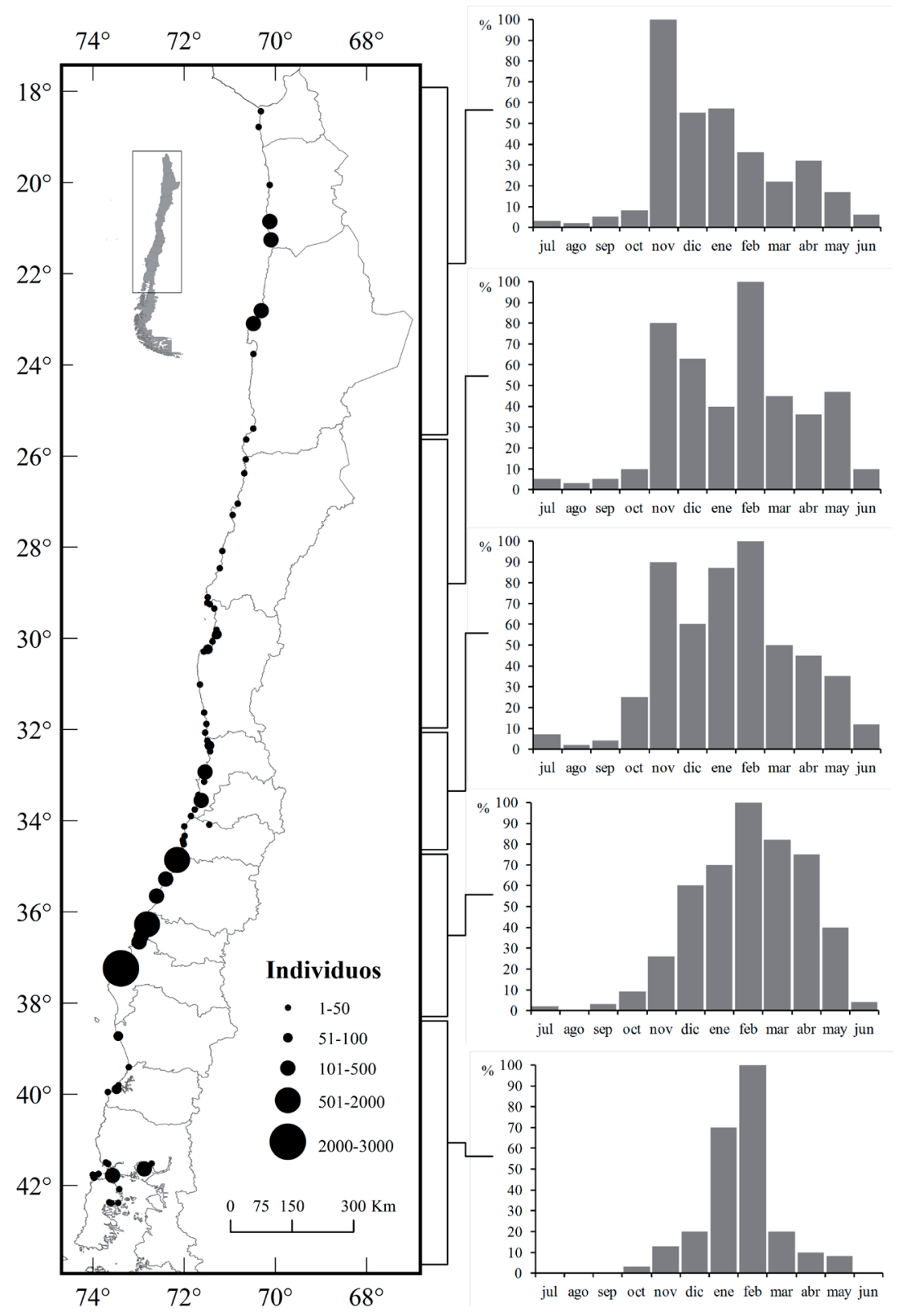

Figura 1. Izquierda: zonas de agregación del rayador (Rynchops niger) en Chile (promedio para período noviembre - abril, entre años 2014 y 2018). Derecha: Abundancia mensual relativa por macrozonas (100\% representa la máxima abundancia promedio para la macrozona). / Left: Black skimmer aggregation zones (Rynchops niger) in Chile (average for the period November - April, between years 2014 and 2018). Right: Relative monthly abundance by macro-zone (100\% represents the maximum average abundance for the macro-zone). 

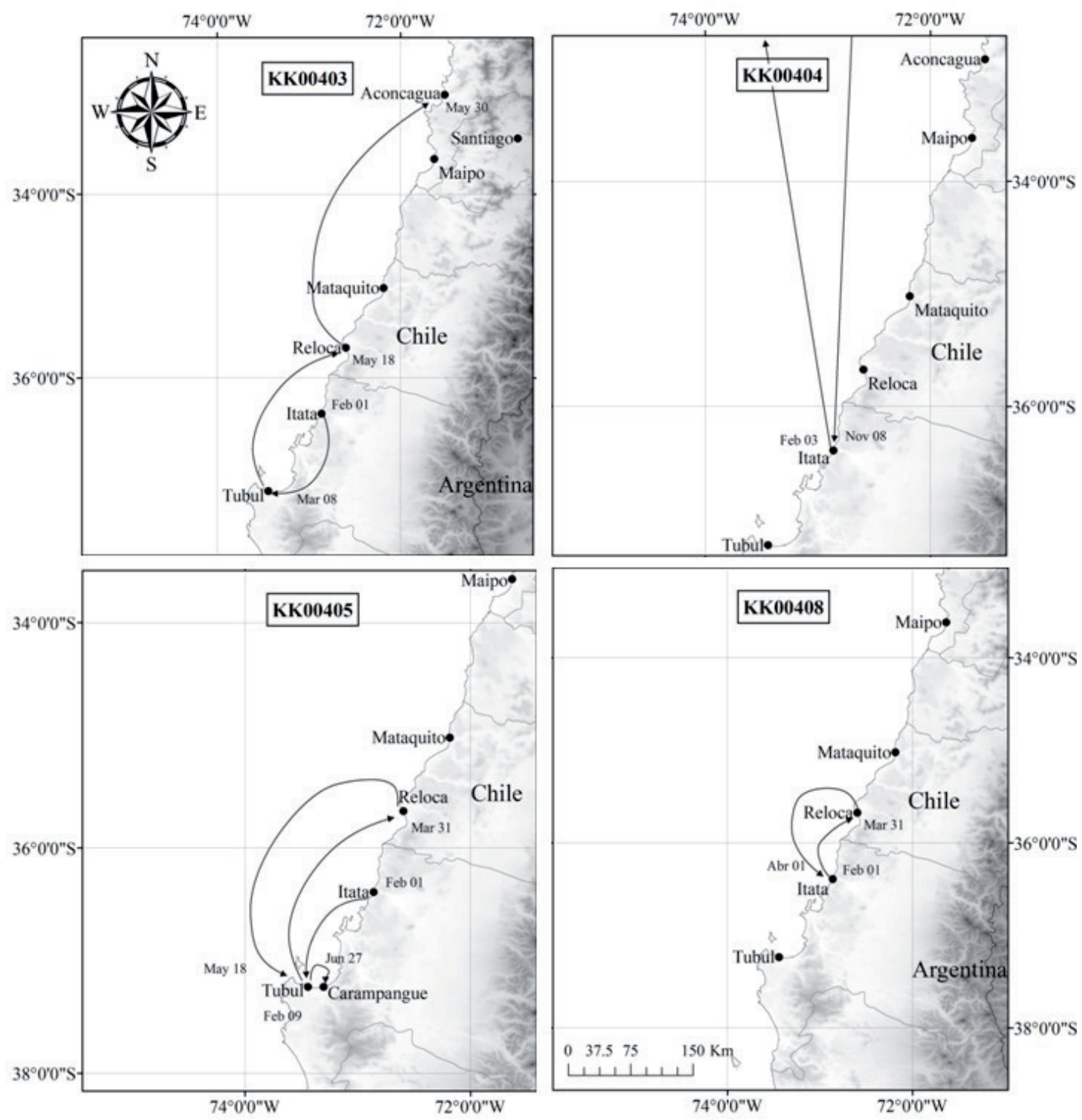

FIGURA 2. Movimientos de cuatro rayadores equipados con radiotransmisores. / Movements of four black skimmers with radiotransmitters attached.

El seguimiento por radiotelemetría mostró el uso de una red de estuarios por parte de los rayadores durante su permanencia estival en la costa pacífica de Sudamérica. Los registros muestran que los desplazamientos entre estuarios pueden ocurrir durante toda la temporada y tanto con dirección norte como sur (Fig. 2). Desplazamientos de 90 km/ noche, como el observado para el individuo KK00408, no deben ser un mayor obstáculo para una especie que puede desplazarse hasta $500 \mathrm{~km}$ en un solo día (Davenport et al. 2016).

Los datos sobre abundancia relativa por mes constituyen evidencia indirecta sobre el movimiento de la especie dentro del país. Por ejemplo, el hecho de que en la zona Norte la máxima abundancia de la especie se observe en noviembre, mes en el cual la especie arriba al país, pero que luego no exista un peak durante el retorno al norte, sugiere que los rayadores pueden usar sitios diferentes durante sus migraciones hacia el sur y hacia el norte. Así, es probable que durante el retorno a sus zonas de reproducción la mayoría de los individuos no se detenga en sitios relativamente importantes en el Norte como la desembocadura del río Lluta, sino que continúen directo a otros humedales, posiblemente en el sur de Perú. En el caso de las macrozonas Centro-Norte y Centro, la existencia de dos peaks sugiere una condición de sitios de paso, donde los 
mayores registros se dan durante la migración al sury el retorno al norte. Finalmente, la presencia de un máximo durante el verano (febrero) en las zonas Centro-Sur y Sur, sugiere que estas regiones representan un destino final para estas aves, lo que coincide con los datos sobre las grandes agregaciones de aves en estuarios de la zona Centro-Sur. Además, el hecho de que el máximo poblacional se alcance en febrero muestra que algunas poblaciones pueden demorar varios meses en llegar desde el extremo norte del país hasta esta zona. Esta estrategia migratoria, con un desplazamiento postreproductivo más lento que la migración pre-reproductiva, es una condición que se observa para especies de diferentes ordenes de aves (Nilsson et al. 2013). Estudios realizados en especies cercanas, como gaviotas y gaviotines, muestran que la migración post-reproductiva tiene una duración del doble de tiempo que la migración pre-reproductiva (Klassen et al. 2011, Egevang et al. 2010).

Aunque ninguno de los individuos marcados permaneció durante el invierno en la zona de estudio, los datos de censo muestran que una pequeña porción de la población permanece en Chile durante la temporada reproductiva. Si bien es cierto, esto no había sido documentado en el país, sí se había reportado para la vertiente atlántica (Vieira et al. 2018).

A diferencia de lo que ocurre en otros países, la casi totalidad de los registros de la especie fueron en sitios costeros, particularmente en estuarios y desembocaduras de ríos, donde el rayador encuentra aguas calmas y poco profundas que requiere para su alimentación (Withers \& Timko 1977). Junto con el hecho de que la costa chilena representa una ruta natural para muchas aves migratorias (Myers et al. 1990), la ausencia de la especie en humedales interiores se debe probablemente a la escasez de ambientes apropiados en un país donde la topografía abrupta sólo permite la existencia de ríos de flujo torrencial (Bauer 2009).

La alta concentración de la especie en unos pocos sitios durante su permanencia en las costas de Chile plantea un desafío evidente para la conservación de esta especie. El hecho de que más del $50 \%$ de la población se congrega en sólo tres sitios, los estuarios de Mataquito, Itata y Tubul (Fig. 1) apoya la condición de estos estuarios como sitios prioritarios para la conservación de aves migratorias (Estades et al. 2012). La alta dependencia de la especie a determinados sitios la hace más vulnerable, ya que la degradación o pérdida de esos sitios podría significar una fuerte disminución del número de individuos o de su sobrevivencia (Iwamura 2013). Por lo tanto, dada la importancia y vulnerabilidad de estos sitios se reconoce en ellos un alto valor estratégico para la conservación de la especie.

\section{AGRADECIMIENTOS}

El seguimiento por radiotelemetría de rayadores y el monitoreo de largo plazo en el que se basó parcialmente este estudio, es financiado por Arauco (programas PREGA, PROMNA y MAPA). Los autores agradecen a Isidora Núñez, Romina Chiappe y Sandra Uribe por su cooperación en la toma de datos. Fernando Medrano amablemente facilitó la base datos de registros de eBird sobre la especie. El Servicio Agrícola y Ganadero del Gobierno de Chile entregó los anillos metálicos con los que se marcaron las aves. Dos revisores anónimos realizaron importantes sugerencias que mejoraron la calidad de este trabajo.

\section{REFERENCIAS}

Acuña, M.P., Vukasovic, M.A., Hernández, H.J., Acuña, T.A., Estades, C.F. 2019. Effects of the surrounding landscape on waterbird populations in estuarine ecosystems of central Chile. Wetlands Ecology and Management 27: 295-310.

Bauer, C.J. 2009. Dams and markets: Rivers and electric power in Chile. Natural Resources Journal 49(3/4): 583-651.

Burger, J. 1982. Jamaica Bay studies: I. Environmental determinants of abundance and distribution of Common Terns (Sterna hirundo) and Black Skimmers (Rynchops niger) at an East Coast estuary. Colonial Waterbirds 5: 148-160.

Clark, R. 1986. Aves de Tierra del Fuego y Cabo de Hornos. Guía de Campo. Ediciones LOLA., Buenos Aires. 294 pp.

Daneri, G., Dellarossa, V., Quiñones, R., Jacob, B., Montero, P., Ulloa, O. 2000. Primary production and community respiration in the Humboldt Current System off Chile and associated oceanic areas. Marine Ecology Progress Series 197: 41-49.

Davenport, L.C., Goodenough, K.S., Haugaasen, T. 2016. Birds of two oceans? Trans-Andean and divergent migration of Black Skimmers (Rynchops niger cinerascens) from the Peruvian Amazon. PloS ONE 11(1): e0144994.

Egevang, C., Stenhouse, I.J., Phillips, R.A., Petersen, A., Fox, J.W., Silk, J.R.D. 2010. Tracking of Arctic Terns Sterna Paradisaea reveals longest animal migration. Proceedings of the National Academy of Sciences of the United States of America 107(5): 2078-2081.

Espinoza, J. 2018. Impacto de perros domésticos sobre aves migratorias en el humedal de Carampangue. Tesis Magister en Áreas Silvestres y Conservación de la Naturaleza. Universidad de Chile, Santiago, Chile.

Estades, C.F., Vukasovic, M.A., Aguirre, J. 2012. Aves en los humedales costeros de Chile. En: Fariña, J.M., Camaño, 
A. (Eds.) Humedales costeros de Chile: Aportes científicos a su gestión sustentable: 67-99. Ediciones Universidad Católica de Chile, Santiago, Chile.

Estades, C.F., Vukasovic, M.A. 2013. Waterbird population dynamics in estuarine wetlands of Central Chile. Ornitología Neotropical 24: 67-83.

Estades, C.F., Vukasovic, M.A., Aguirre, J. 2017. Birds in Coastal Wetlands of Chile. En: Fariña, J.M., Camaño, A. (Eds.) The Ecology and Natural History of Chilean Saltmarshes: 4770. Springer, Cham.

González, A.L., Vukasovic, M.A., Estades, C.F. 2011. Variación temporal en la abundancia y diversidad de aves en el humedal del río Itata, región del Bío-Bío, Chile. Gayana 75(2): 170-181.

Groom, M.J. 1992. Sand-colored Nighthawks parasitize the antipredator behavior of three nesting bird species. Ecology 73(3): 785-793.

Hajek, E., Di Castri, F. 1975. Bioclimatografía de Chile. Editorial Universidad Católica de Chile, Santiago. 107 pp.

Horns, J.J., Adler, F.R., Şekercioğlu, Ç.H. 2018. Using opportunistic citizen science data to estimate avian population trends. Biological conservation 221: 151-159.

Iwamura, T., Possingham, H.P., Chadès, I., Minton, C., Murray, N.J., Rogers, D.I., Treml, E.A., Fuller, R.A. 2013. Migratory connectivity magnifies the consequences of habitat loss from sea-level rise for shorebird populations. Proceedings of the Royal Society B: Biological Sciences 280: 1-8.

Klaassen, R.H.G., Ens, B.J., Shamoun-Baranes, J., Exo, K.-M., Bairlein, F. 2011. Migration strategy of a flight generalist, the Lesser Black-backed Gull Larus fuscus. Behavioral Ecology 23 (1): 58-68.

Myers, J.P., Sallaberry, A.M., Ortiz, E., Castro, G., Gordon, L.M., Maron, J.L., Schick, C.T., Tabilo, E., Antas, P., Below, T. 1990. Migration routes of new world sanderlings (Calidris alba). The Auk 107(1): 172-180.

Nilsson, C., Klaassen, R.H., Alerstam, T. 2013. Differences in speed and duration of bird migration between spring and autumn. The American Naturalist 181(6): 837-845.

Sallaberry, M., Tabilo, E., Klesse, C., Abarca, J. 1996. The Chilean Shorebird Network (RECAP). International Wader Studies 8: 71-78.

Soazo, P.O., Jorquera, I.R., Garrido, P.A., Jaramillo, Í. 2009. Important Bird Areas AMERICAS. URL: http://datazone. birdlife.org/userfiles/file/IBAs/AmCntryPDFs/Chile.pdf

Sullivan, B.L., Aycrigg, J.L., Barry, J.H., Bonney, R.E., Bruns, N., Cooper, C.B., Damoulas, T., Dhondt, A.A., Dietterich, T., Farnsworth, A., Fink, D., Fitzpatrick, J.W., Fredericks, T., Gerbracht, J., Gomes, C., Hochachka, W.M., Iliff, M.J., Lagoze, C., La Sorte, F.A., Merrifield, M., Morris, W., Phillips, T.B., Reynolds, M., Rodewald, A.D., Rosenberg, K.V., Trautmann, N.M., Wiggins, A., Winkler, D.W., Wong, W.K., Wood, C.L., Yu, J., Kelling, S. 2014. The eBird enterprise: An integrated approach to development and application of citizen science. Biological Conservation 169: 31-40.

Tomkins, I.T. 1951. Method of Feeding of the Black Skimmer, Rynchops nigra. The Auk 68: 236-239

Vieira, B.P., Furness, R.W., Nager, R.G. 2018. What do we know about Black Skimmers? A review on its annual-cycle and life-history main events. Ardea 106(2): 119-130.

Wetmore, A. 1944. The subspecific characters and distribution of the New World Skimmers - (Rynchops nigra). Caldasia 3(11): 111-118.

Withers, P.C., Timko, P.L. 1977. The significance of ground effect to the aerodynamic cost of flight and energetics of the black skimmer (Rhyncops nigra). Journal of Experimental Biology 70(1): 13-26.

Zusi, R.L. 1962. Structural adaptations of the head and neck in the Black Skimmer. Publications of the Nuttall Ornithological Club, Cambridge: The Club.

Zusi, R.L., Bridge, D. 1981. On the slit pupil of the black skimmer (Rynchops niger). Journal of Field Ornithology 52(4): 338340.

Received: 02.04.2020

Accepted: 27.08.2020 